\title{
PARTIAL DIFFERENTIAL EQUATIONS WITH PIECEWISE CONSTANT DELAY
}

\author{
JOSEPH WIENER \\ Department of Mathematics \\ The University of Texas - Pan American \\ Edinburg, Texas 78539 \\ and \\ LOKENATH DEBNATH \\ Department of Mathematics \\ University of Central Florida \\ Orlando, Florida 32816
}

(Received October 25, 1990 and in revised form November 23, 1990)

\begin{abstract}
The influence of certain discontinuous delays on the behavior of solutions to partial differential equations is studied. In Section 2, the initial value problems (IVP) are discussed for differential equations with piecewise constant argument (EPCA) in partial derivatives. A class of loaded partial differential equations that arise in solving certain inverse problems is studied in some detail in Section 3. Section 4 is devoted to obtain the solutions of IVP for linear partial differential equations with piecewise constant delay by using integral transforms. Finally, an abstract Cauchy problem is discussed.
\end{abstract}

KEY WORDS AND PHRASES. Partial Differential Equation, Piecewise Constant Delay, Loaded Equation, Initial Value Problem, Existence, Uniqueness, Abstract Cauchy Problem.

1980 AMS Subject Classification Codes. 35A05, 35B25, 35L10, 34K25.

\section{INTRODUCTION.}

Functional differential equations (FDE) with delay provide a mathematical model for a physical or biological system in which the rate of change of the system depends upon its past history. The theory of FDE with continuous argument is well developed and has numerous applications in natural and engineering sciences. This paper continues our earlier work [1-5] in an attempt to extend this theory to differential equations with discontinuous argument deviations. In these papers, ordinary differential equations with arguments having intervals of constancy have been studied. Such equations represent a hybrid of continuous and discrete dynamical systems and combine properties of both differential and difference equations. They include as particular cases loaded and impulse equations, hence their importance in control theory and in certain biomedical models. Continuity of a solution at a point joining any two consecutive intervals implies recursion relations for the values of the solution at such points. Therefore, differential equations with piecewise constant argument (EPCA) are intrinsically closer to difference rather than differential equations. In [6] boundary value problems for some linear EPCA in partial derivatives were considered and the behavior of their solutions studied. The results were also extended to equations with positive definite 
operators in Hilbert spaces [7]. Here initial value problems (IVP) are studied for EPCA in partial derivatives. A class of loaded equations that arise in solving certain inverse problems is explored within the general framework of differential equations with piecewise constant delay.

\section{INITIAL VALUE PROBLEMS.}

It has been shown in [6] that partial differential equations (PDE) with piecewise constant time naturally arise in the process of approximating PDE by simpler EPCA. Thus, if in the equation

$$
u_{t}=a^{2} u_{x x}-b u \text {, }
$$

which describes heat flow in a rod with both diffusion $a^{2} u_{x x}$ along the rod and heat loss (or gain) across the lateral sides of the rod, the lateral heat change is measured at discrete moments of time, then we get an equation with piecewise constant argument

$$
u_{t}(x, t)=a^{2} u_{x x}(x, t)-b u(x, n h),
$$

where $t \in[n h,(n+1) h], n=0,1, \ldots$ and $h>0$ is some constant. This equation can be written in the form

$$
u_{t}(x, t)=a^{2} u_{x x}(x, t)-b u(x,[t / h] h),
$$

where $[\cdot]$ designates the greatest integer function. Ordinary differential equations with arguments $[t],[t-n],[t+n]$ have been investigated in $[1-4]$, with $[t+1 / 2]$ in [5], and with $[t / h] h$ in [8-9]. Furthermore, EPCA have been used recently in [9] to approximate solutions of equations with continuous delay. The diffusion-convection equation

$$
u_{t}=a^{2} u_{x x}-c u_{x}
$$

describes, for instance, the concentration $u(x, t)$ of a pollutant carried along in a stream moving with velocity $c$. The term $a^{2} u_{x x}$ is the diffusion contribution and $-c u_{x}$ is the convection component. If the convection part is measured at discrete times $n h$, the process results in the equation

$$
u_{t}(x, t)=a^{2} u_{x x}(x, t)-c u_{x}(x,[t / h] h) .
$$

We consider the initial value problem (IVP)

$$
\begin{aligned}
\frac{\partial u(x, t)}{\partial t}+P\left(\frac{\partial}{\partial x}\right) u(x, t) & =Q\left(\frac{\partial}{\partial x}\right) u(x,[t / h] h), \\
u(x, 0) & =u_{0}(x),
\end{aligned}
$$

where $P$ and $Q$ are polynomials of the highest degree $m$ with constant coefficients, [•] designates the greatest integer function, $h=$ const $>0$, and

$$
(x, t) \in G=(-\infty, \infty) \times[0, \infty) .
$$

DEFINITION 2.1. A function $u(x, t)$ is called a solution of IVP (2.6) if it satisfies the conditions: (i) $u(x, t)$ is continuous in $G$; (ii) $\partial u / \partial t$ and $\partial^{k} u / \partial x^{k}(k=0,1 \ldots, m)$ exist and are continuous in $G$, with the possible exception of the points $(x, n h)$, where one-sided derivatives exist $(n=0,1,2 .$.$) ; (iii) u(x, t)$ satisfies Eq. (2.6) in $G$, with the possible exception of the points $(x, n h)$, and the initial condition $u(x, 0)=u_{0}(x)$.

Let $u_{n}(x, t)$ be the solution of the given problem on the interval $n h \leq t<(n+1) h$, then

$$
\partial u_{n}(x, t) / \partial t+P u_{n}(x, t)=Q u_{n}(x),
$$

where

$$
u_{n}(x)=u_{n}(x, n h)
$$


Write

$$
u_{n}(x, t)=w_{n}(x, t)+v_{n}(x),
$$

which gives the equation

$$
\partial w_{n} / \partial t+P w_{n}+P v_{n}(x)=Q u_{n}(x),
$$

and require that

$$
\begin{gathered}
\partial w_{n} / \partial t+P w_{n}=0, \\
P v_{n}(x)=Q u_{n}(x) .
\end{gathered}
$$

Let $v_{n}(x)$ be a solution of $\operatorname{ODE}(2.11)$, then at $t=n h$ we have

$$
w_{n}(x, n h)=u_{n}(x)-v_{n}(x),
$$

and it remains to consider Eq. (2.10) with initial condition (2.12). It is well known that the solution $E(x, t)$ of the problem

$$
\partial w / \partial t+P w=0, w(x, 0)=w_{0}(x)
$$

with $w_{0}(x)=\delta(x)$, where $\delta(x)$ is the Dirac delta functional, is called its fundamental solution. The solution of IVP (2.13) is given by the convolution

$$
w(x, t)=E(x, t) * w_{0}(x) .
$$

Hence, the solution of problem (2.10)-(2.12) can be written as

$$
w_{n}(x, t)=E(x, t-n h) * w_{n}(x, n)
$$

and the solution of (2.7), (2.8) is

$$
\begin{gathered}
u_{n}(x, t)=E(x, t-n h) *\left(u_{n}(x)-v_{n}(x)\right)+v_{n}(x), \\
(n h \leq t<(n+1) h) .
\end{gathered}
$$

Continuity of the solution at $t=(n+1) h$ implies

$$
u_{n}(x,(n+1) h)=u_{n+1}(x,(n+1) h)=u_{n+1}(x),
$$

that is,

$$
u_{n+1}(x)=E(x, h) *\left(u_{n}(x)-v_{n}(x)\right)+v_{n}(x) .
$$

Formulas (2.16), (2.17) successively determine the solution of IVP (2.6) on each interval $\boldsymbol{n} \boldsymbol{h} \boldsymbol{t} \mathbf{s}(\boldsymbol{n}+1) \boldsymbol{h}$. Indeed, from $P v_{0}(x)=Q u_{0}(x)$ we find $v_{0}(x)$ and substitute both $u_{0}(x)$ and $v_{0}(x)$ in (2.16) and (2.17), to obtain $u_{0}(x, t)$ and $u_{1}(x)$. Then we use $u_{1}(x)$ in (2.11) to find $v_{1}(x)$ and substitute $u_{1}(x)$ and $v_{1}(x)$ in (2.16) and (2.17), which yields $u_{1}(x, t)$ and $u_{2}(x)$. Continuing this procedure leads to $u_{n}(x, t)$, the solution of (2.6) on $[n h,(n+1) h]$. The solution $v_{n}(x)$ of $(2.11)$ is defined to within an arbitrary polynomial $q(x)$ of degree $<m$. Since $q(x)$ is a solution of Eq. (2.13) with the initial condition $w(x, 0)=q(x)$, then $q(x)=E(x, t) * q(x)$ and $q(x)$ cancels in formulas (2.16), (2.17). This concludes the proof of the following assertion.

THEOREM 2.1. If Eq. (2.13) with $w(x, 0)=u_{0}(x)$ has a unique solution on $t \in(0, \infty)$, then there exists a unique solution of IVP $(2.6)$ on $(0, \infty)$ and it is given by $(2.16)$, for each interval $n h \leq t \leq(n+1) h$.

COROLLARY 2.1. There exist unique solutions of Eqs. (2.3) and (2.5), with $u(x, 0)=u_{0}(x)$, in the class of functions that grow to infinity slower than $\exp \left(x^{2}\right)$ as $|x| \rightarrow \infty$.

For Eqs. (2.3) and (2.5) we have

$$
v_{n}(x)=a^{-2} b \int_{0}^{x}(x-s) u_{n}(s) d s \text { and } v_{n}(x)=a^{-2} c \int_{0}^{x} u_{n}(s) d s,
$$


respectively, and $E(x, t)=\exp \left(-x^{2} / 4 a^{2} t\right) / 2 a \sqrt{\pi t}$.

Formula (2.16) for the solution of the problem

$$
u_{t}(x, t)=a^{2} u_{x x}(x, t)-b u_{x x}(x,[t / h] h), u(x, 0)=u_{0}(x)
$$

on $n h \leq t<(n+1) h$ becomes

$$
u_{n}(x, t)=\left(1-\frac{b}{a^{2}}\right) E(x, t-n h) * u_{n}(x)+\frac{b}{a^{2}} u_{n}(x),
$$

where $E(x, t)$ is the same as in Eqs. (2.3) and (2.5).

The above method may also be used to solve IVP for PDE of any order in $t$ with piecewise constant delay or systems of such equations. In the latter case, $P$ and $Q$ in (2.6) are square matrices of linear differential operators and $u(x, t)$ is a vector function. Thus, the solution $u_{n}(x, t)$ of the problem

$$
\begin{gathered}
u_{t t}(x, t)=a^{2} u_{x x}(x, t)-b u_{x x}(x,[t]), \\
u(x, 0)=f_{0}(x), u_{t}(x, 0)=g_{0}(x)
\end{gathered}
$$

on $n \leq t<n+1$ is sought in the form $u_{n}(x, t)=w_{n}(x, t)+v_{n}(x)$ whence $a^{2} v_{n}{ }^{\prime \prime}(x)-b u_{n}{ }^{\prime \prime}(x, n)=0$ and $\partial^{2} w_{n} / \partial t^{2}=a^{2} \partial^{2} w_{n} / \partial x^{2}$. Setting $u(x, n)=f_{n}(x), \quad u_{t}(x, n)=g_{n}(x)$ gives $v_{n}(x)=a^{-2} b f_{n}(x)$, $w(x, n)=\left(1-a^{-2} b\right) f_{n}(x), w_{t}(x, n)=g_{n}(x)$, and

$$
\begin{aligned}
u_{n}(x, t)=\frac{b}{a^{2}} f_{n}(x) & +\left(1-\frac{b}{a^{2}}\right) \frac{f_{n}(x-a(t-n))+f_{n}(x+a(t-n))}{2} \\
& +\frac{1}{2 a} \int_{x=a(t-n)}^{x+a(t-n)} g_{n}(s) d s .
\end{aligned}
$$

Putting $t=n+1$ produces the recursion relations

$$
\begin{aligned}
f_{n+1}(x)=\frac{b}{a^{2}} f_{n}(x) & +\left(1-\frac{b}{a^{2}}\right)\left[\frac{f_{n}(x-a)+f_{n}(x+a)}{2}\right] \\
& +\frac{1}{2 a} \int_{x-a}^{x+a} g_{n}(s) d s, \\
g_{n+1}(x) & =\left(1-\frac{b}{a^{2}}\right) \frac{a f_{n}^{\prime}(x+a)-a f_{n}^{\prime}(x-a)}{2} \\
& +\frac{1}{2}\left(g_{n}(x+a)+g_{n}(x-a)\right) .
\end{aligned}
$$

\section{LOADED EOUATIONS.}

Loaded partial differential equations have properties similar to those of equations with piecewise constant delay. The IVP for the following class of loaded equations

$$
\begin{aligned}
\frac{\partial u(x, t)}{\partial t} & =P\left(\frac{\partial}{\partial x}\right) u(x, t)+\sum_{j=1}^{q} Q_{j}\left(\frac{\partial}{\partial x}\right) u\left(x, t_{j}\right), \\
u(x, 0) & =u_{0}(x)
\end{aligned}
$$


was considered in [10], where $(x, t) \in R^{n} \times[0, T]$, the $t, \in(0, T]$ are given, $P(s)$ and $Q,(s)$ are polynomials in $s=\left(s_{1}, \ldots, s_{n}\right)$, and $\sum\left|Q_{j}(s)\right| \equiv 0$. Eq. (3.1) arises in solving certain inverse problems for systems with elements concentrated at specific moments of time. The Fourier transform $U(s, t)$ of $u(x, t)$ satisfies the equation

$$
U_{t}(s, t)=P(i s) U(s, t)+\sum_{j=1}^{q} Q_{j}(i s) U\left(s, t_{j}\right)
$$

whence

$$
U(s, t)=U_{0}(s) e^{P(s k}+k(P(i s), t) \sum_{j=1}^{q} Q_{j}(i s) U\left(s, t_{j}\right),
$$

where $U_{0}(s)$ is the Fourier transform of $u_{0}(x)$ and

$$
k(P(i s), t)=\int_{0}^{t} e^{P(1 s) y} d y .
$$

Denote

$$
A_{J}=U_{0}(s) e^{P(i s)_{j}}, k_{j}=k\left(P(i s), t_{j}\right), B=\sum_{j=1}^{q} Q_{j}(i s) U\left(s, t_{j}\right)
$$

then multiply by $Q_{,}(i s)$ each of the equations

$$
U\left(s, t_{j}\right)=A_{j}+k_{j} B, j=1, \ldots, q
$$

and add them. Hence,

$$
B=\sum_{j=1}^{q} A, Q,(i s)+B \sum_{j=1}^{q} k_{j} Q_{j}(i s)
$$

or

$$
\left(1-\sum_{j=1}^{q} k_{j} Q_{j}(i s)\right) B=\sum_{j=1}^{q} A_{j} Q_{j}(i s) .
$$

The equation

$$
\Delta(s) \equiv 1-\sum_{j=1}^{q} Q_{j}(i s) k\left(P(i s), t_{j}\right)=0
$$

is called the characteristic equation for (3.1) and its solution set $Z$ is called the characteristic variety of (3.1). It is said [10] that (3.1) is absolutely non-degenerate if $Z=\varnothing$, non-degenerate of type $a$ if $a=\inf |\operatorname{Ims}|<\infty, s \in Z \neq C^{n}$, and degenerate if $Z=C^{n}$. The case $Z=\varnothing$ implies $\Delta(s)=$ const, since $\Delta(s)$ is meromorphic, and a meromorphic function that is not constant assumes every complex value with at most two exceptions. The equation $\Delta(s)=C$ can be written as

$$
P(i s)+\sum_{j=1}^{q} Q_{j}(i s)-\sum_{j=1}^{q} Q_{j}(i s) e^{P(i s) t,}=C P(i s)
$$

and is possible for $q>1$ only if $P(s)=$ const, otherwise $\exp \left(P(i s) t_{j}\right)$ would grow faster than any polynomial, which breaks the latter equality. For $q=1$, we have

$$
\Delta(s)=\left(P(i s)+Q_{1}(i s)-Q_{1}(i s) e^{P(i s) t_{1}}\right) / P(i s),
$$

and in this case $Z=\varnothing$ is equivalent to $P(i s)+Q(i s) \equiv 0$. On the other hand, $\Delta(s)=0$ is equivalent to

$$
P(i s)+\sum_{j=1}^{q} Q_{j}(i s)-\sum_{j=1}^{q} Q_{j}(i s) e^{P(i s) t}=0 \text {, }
$$


which implies $P(s)=$ const. This establishes the following proposition which was stated in [10] without proof.

LEMMA 3.1. Eq. (3.1) is absolutely non-degenerate if only if either of the following conditions holds true:

(i) $P(s) \equiv C_{1}, \sum_{j=1}^{q} Q_{j}(s) k\left(C_{1}, t_{j}\right) \equiv C_{2} \neq 1$;

or

(ii) $\quad q=1, P(s)+Q_{1}(s) \equiv 0$.

Eq. (3.1) is degenerate if and only if

$$
P(s) \equiv C_{1}, \sum_{j=1}^{q} Q_{j}(s) k\left(C_{1}, t_{j}\right) \equiv 1 .
$$

Substituting $B$ from (3.8) in (3.4) leads to the proof of the following theorems which were formulated in [10].

THEOREM 3.1. The uniqueness classes for the solution of the Cauchy problem for an absolutely non-degenerate equation (3.1) are the same as those for the equation (without "loads") $u_{t}(x, t)=P u(x, t)$.

THEOREM 3.2. The homogeneous degenerate IVP $(3.1)\left(u_{0}(x)=0\right)$ has non-trivial solutions, with compact support.

THEOREM 3.3. Suppose that Eq. (3.1) is of finite type $a(0<a<\infty)$ and that $u(x, t)$ is a solution of $(3.1)$ with $u_{0}(x) \equiv 0$. If

$$
|u(x, t)| \leq C e^{\alpha|x|}, x \in R^{n}, t \in[0, T],
$$

and $\alpha<a$, then $u(x, t) \equiv 0$. For any $\alpha>a$ there exists a solution $u(x, t) \neq 0$ of $(3.1)$ with $u_{0}(x)=0$ satisfying (3.13).

The uniqueness classes for the solution of the Cauchy problem for the equation $u_{t}(x, t)=P u(x, t)$ were explored in [11] and consist of the functions that grow no faster than $\exp \left(a|x|^{\alpha}\right)$ as $|x| \rightarrow \infty$, where $\alpha>1$ depends on the degree of $P(s)$. Integral transformations can be used also in the study of EPCA.

\section{SOLUTION FORMULAS.}

The purpose of this section is to show that integral transforms can be successfully used to find the solutions of IVP for linear partial differential equations with piecewise constant delay.

THEOREM 4.1. The solution of the problem

$$
u_{t}(x, t)=a^{2} u_{x x}(x, t)-b u_{x x}(x,[t]), \quad u(x, 0)=u_{0}(x)
$$

is given by the formula

$$
\begin{aligned}
u(x, t)=\sum_{j=0}^{[t]}\left(\begin{array}{c}
{[t]} \\
j
\end{array}\right) & \left(1-\frac{b}{a^{2}}\right)^{j}\left(\frac{b}{a^{2}}\right)^{[t-j]}\left(\frac{b}{a^{2}} E(x, j)\right. \\
& \left.+\left(1-\frac{b}{a^{2}}\right) E(x, t+j-[t])\right) * u_{0}(x),
\end{aligned}
$$

where $E(x, t)=\exp \left(-x^{2} / 4 a^{2} t\right) / 2 a \sqrt{\pi t}$ and $E(x, 0) * u_{0}(x)=u_{0}(x)$.

PROOF. For $n \leq t<n+1$, Eq. (4.1) becomes

$$
u_{t}(x, t)=a^{2} u_{x x}(x, t)-b u_{x x}(x, n),
$$


and the Fourier transform $U(\omega, t)=F(u(x, t))$ satisfies the equation

$$
U_{(}(\omega, t)=-a^{2} \omega^{2} U(\omega, t)+b \omega^{2} U(\omega, n),
$$

whence

$$
U(\omega, t)=C e^{-a^{2} \omega^{2}(t-n)}+\frac{b}{a^{2}} U(\omega, n) .
$$

At $t=n$ we have

$$
U(\omega, n)=C+\frac{b}{a^{2}} U(\omega, n), C=\left(1-\frac{b}{a^{2}}\right) U(\omega, n),
$$

and

$$
U(\omega, t)=\left(\frac{b}{a^{2}}+\left(1-\frac{b}{a^{2}}\right) e^{-a^{2} \omega^{2}(t-n)}\right) U(\omega, n) .
$$

At $t=n+1$ this gives

$$
U(\omega, n+1)=\left(\frac{b}{a^{2}}+\left(1-\frac{b}{a^{2}}\right) e^{-a^{2} \omega^{2}}\right) U(\omega, n)
$$

and

$$
U(\omega, n)=\left(\frac{b}{a^{2}}+\left(1-\frac{b}{a^{2}}\right) e^{-a^{2} \omega^{2}}\right)^{n} U(\omega, 0)
$$

Substituting the binomial expansion of $U(\omega, n)$ in (4.7) yields (4.2).

THEOREM 4.2. The solution of Eq. (2.1) with the initial condition $u(x, 0)=u_{0}(x)$ is given by the formula

$$
u(x, t)=u_{0}(x) *\left(F_{x x}(x, t)-\frac{b}{a^{2}} F(x, t)+\frac{b}{a^{2}} F(x, h[t / h]),\right.
$$

where

$$
F(x, t)=\sum_{j=0}^{[t / h]}\left(\begin{array}{c}
{[t / h]} \\
j
\end{array}\right) \frac{b^{j}}{a^{2 j}} \sum_{k=0}^{j}\left(\begin{array}{l}
j \\
k
\end{array}\right)(-1)^{k} E(x, t-(j-k) h) * \frac{x^{2 j+1}}{(2 j+1) !} H(x),
$$

$H(x)=1$, for $x>0$, and $H(x)=0$, for $x<0$.

PROOF. For $n h \leq t<(n+1) h$, we have

$$
u_{t}(x, t)=a^{2} u_{x x}(x, t)-b u(x, n h),
$$

and the two-sided Laplace transform $U(s, t)=L(u(x, t))$ satisfies the equation

$$
U_{t}(s, t)=a^{2} s^{2} U(s, t)-b U_{n}(s), U_{n}(s)=U(s, n h)
$$

whence

$$
U(s, t)=\left(e^{a^{2} s^{2}(t-n h)}\left(1-\frac{b}{a^{2} s^{2}}\right)+\frac{b}{a^{2} s^{2}}\right) U_{n}(s) .
$$

At $t=(n+1) h$ this gives

$$
U_{n+1}(s)=\left(e^{a^{2} h s^{2}}\left(1-\frac{b}{a^{2} s^{2}}\right)+\frac{b}{a^{2} s^{2}}\right) U_{n}(s)
$$

and 


$$
\begin{gathered}
U_{n}(s)=\left(e^{a^{2} h s^{2}}+\left(1-e^{a^{2} h s^{2}}\right) \frac{b}{a^{2} s^{2}}\right)^{n} U_{0}(s), \\
U_{0}(s)=L\left(u_{0}(x)\right) .
\end{gathered}
$$

Hence

$$
U_{n}(s)=U_{0}(s) \sum_{j=0}^{n}\left(\begin{array}{l}
n \\
j
\end{array}\right) \frac{b^{j}}{a^{2} s^{2}} \sum_{k=0}^{j}\left(\begin{array}{l}
j \\
k
\end{array}\right)(-1)^{k} e^{a^{2} h s^{2}(n+k-j)}
$$

and

$$
\begin{array}{r}
U(s, t)=U_{0}(s) \sum_{j=0}^{n}\left(\begin{array}{l}
n \\
j
\end{array}\right) \frac{b^{j}}{a^{2 j} s^{2 j}} \sum_{k=0}^{j}\left(\begin{array}{l}
j \\
k
\end{array}\right)(-1)^{k}\left(e^{a^{2} s^{2}(t-(j-k) h)}\right. \\
\left.\frac{b}{a^{2} s^{2}} e^{a^{2} s^{2}(t-(j-k) h)}+\frac{b}{a^{2} s^{2}} e^{a^{2} h s^{2}(n+k-j)}\right),
\end{array}
$$

which proves the result.

THEOREM 4.3. The solution of Eq. (2.5) with $u(x, 0)=u_{0}(x)$ is given by the formula

$$
u(x, t)=u_{0}(x) *\left(F_{x x}(x, t)-\frac{c}{a^{2}} F_{x}(x, t)+\frac{c}{a^{2}} F_{x}(x, h[t / h]),\right.
$$

where $F(x, t)$ is defined in (4.11).

THEOREM 4.4. The solution of problem (2.6) is given by the formula

$$
\begin{aligned}
u(x, t)=u_{0}(x) & * \sum_{j=0}^{[t / h]}\left(\begin{array}{c}
{[t / h]} \\
j
\end{array}\right)\left(Q^{j}(\partial / \partial x) P_{j}(x) * F_{j}(x, t)\right. \\
& +Q^{j+1}(\partial / \partial x) P_{j+1}(x) *\left(F_{j}(x, h[t / h])-F_{j}(x, t)\right),
\end{aligned}
$$

where

$$
F_{j}(x, t)=\sum_{k=0}^{j}\left(\begin{array}{l}
j \\
k
\end{array}\right)(-1)^{k} E(x, t-(j-k) h),
$$

$E(x, t)$ is the fundamental solution of $(2.13)$, and $P_{j}(x)$ is the inverse Laplace transform of $P^{j}(s)$.

PROOF. The solution $u_{n}(x, t)$ of (2.6) on the interval $n h \leq t<(n+1) h$ satisfies (2.7) - (2.8), and for its two-sided Laplace transform in $x$ we obtain the equation

$$
U_{t}(s, t)+P(s) U(s, t)=Q(s) U_{n}(s), U_{n}(s)=U(s, n),
$$

whence

$$
U(s, t)=C e^{-P(s)(t-n k)}+P^{-1}(s) Q(s) U_{n}(s) .
$$

At $t=n h$ we have

$$
U_{n}(s)=C+P^{-1}(s) Q(s) U_{n}(s)
$$

and

$$
U(s, t)=\left(e^{-P(s)(t-n h)}+\left(1-e^{-P(s)(t-n h)}\right) P^{-1} Q\right) U_{n}(s) .
$$

At $t-(n+1) h$ this gives

$$
U_{n+1}(s)=\left(e^{-P(s) h}+\left(1-e^{-P(s) h}\right) P^{-1} Q\right) U_{n}(s),
$$

hence,

$$
U_{n}(s)=\left(e^{-P(s) h}+\left(1-e^{-P(s) h}\right) P^{-1} Q\right)^{n} U_{0}(s)
$$


and

$$
U_{n}(s)=U_{0}(s) \sum_{j=0}^{n}\left(\begin{array}{l}
n \\
j
\end{array}\right) Q^{j} P^{-j} \sum_{k=0}^{j}\left(\begin{array}{l}
j \\
k
\end{array}\right)(-1)^{k} e^{-P(s) h(n-j+k)}
$$

Therefore,

$$
\begin{aligned}
U(s, t) & =\sum_{j=0}^{n}\left(\begin{array}{l}
n \\
j
\end{array}\right) Q^{j} P^{-j} \sum_{k=0}^{j}\left(\begin{array}{l}
j \\
k
\end{array}\right)(-1)^{k} e^{-P(s)(t-(j-k) h)} \\
& +\sum_{j=0}^{n}\left(\begin{array}{l}
n \\
j
\end{array}\right) Q^{j+1} P^{-\jmath-1} \sum_{k=0}^{j}\left(\begin{array}{l}
j \\
k
\end{array}\right)(-1)^{k} e^{-P(s) h(n-j+k)} \\
& -\sum_{j=0}^{n}\left(\begin{array}{l}
n \\
j
\end{array}\right) Q^{j+1} P^{-\jmath-1} \sum_{k=0}^{j}\left(\begin{array}{l}
j \\
k
\end{array}\right)(-1)^{k} e^{-P(s)(t-(j-k) h)},
\end{aligned}
$$

which leads to (4.21).

Linear differential equations in Banach space with arguments $[t]$ and $t-n[t]$ have been studied in [2]. Consider in a Banach space $Y$ the equation

$$
u^{\prime}(t)=A u(t)+B u([t])
$$

with linear constant operators $A: D(A) \rightarrow Y$ and $B: D(B) \rightarrow Y$, their domains $D(A) \subset D(B) \subset Y$, and $D(A)$ is everywhere dense in $Y$. According to [2], a solution of Eq. (4.31) on $[0, \infty)$ is a function $u(t)$ satisfying the conditions: (i) $u(t)$ is continuous on $[0, \infty)$ and its values lie in the domain $D(A)$ for all $t \in[0, \infty)$. (ii) At each point $t \in[0, \infty)$ there exists a strong derivative $u^{\prime}(t)$, with the possible exception of the points $[t] \in[0, \infty)$ where one-sided derivatives exist. (iii) Eq. (4.31) is satisfied on each interval $[n, n+1) \subset[0, \infty)$ with integral endpoints. The Cauchy problem on $[0, \infty)$ is to find a solution of the equation on $[0, \infty)$ satisfying the initial condition

$$
u(0)=u_{0} \in D(A) \text {. }
$$

The properties of solutions to Eq. (4.31) with bounded operators are similar to those of solutions to systems of ordinary differential equations which can be viewed as equations in a finite-dimensional Banach space. Indeed, if $A, B: Y \rightarrow Y$ are bounded linear operators and $A$ is bijective, then problem (4.31) - (4.32) on $[0, \infty)$ has a unique solution [2]

$$
u(t)=V(t-[t]) V^{[t]}(1) u_{0}
$$

where

$$
V(t)=e^{A t}+\left(e^{A t}-I\right) A^{-1} B .
$$

This solution cannot grow to infinity faster than exponentially. If, in addition, there exists a bounded inverse of the operator $V(1)$, then the solution has a unique backward continuation on $(-\infty, 0]$ given by formula (4.33). The Cauchy problem

$$
u^{\prime}(t)=A u(t), \quad u(0)=u_{0} \in D(A)
$$

is correctly posed on $[0, \infty)$ if for any $u_{0} \in D(A)$ it has a unique solution, and this solution depends continuously on the initial data in the sense that if $u_{n}(0) \rightarrow 0\left(u_{n}(0) \in D(A)\right)$, then $u_{n}(t) \rightarrow 0$ for the corresponding solution at every $t \in[0, \infty)$. If the Cauchy problem (4.35) is correct, its solution is given by the formula

$$
u(t)=T(t) u_{0} \quad\left(u_{0} \in D(A)\right),
$$


where $T(t)$ is a semigroup of strongly continuous operators for $t>0$. For many applications it is necessary to extend the concept of solution of the Cauchy problem. A weakened solution of Eq. $(4.35)$ on $[0, \infty)$ is a function $u(t)$ which is continuous on $[0, \infty)$, strongly continuously differentiable on $(0, \infty)$ and satisfies the equation there. By a weakened Cauchy problem on $[0, \infty)$ we mean the problem of finding a weakened solution satisfying the initial condition $u(0)=u_{0}$. Here the element $u_{0}$ may already not lie in the domain of the operator $\boldsymbol{A}$. Thus, the demands on the behavior of the solution at $t=0$ are relaxed. On the other hand, we require the continuity of the derivative of the solution for $t>0$. However, for a correct Cauchy problem this requirement is automatically satisfied. The following result has been proved in [2].

THEOREM 4.5. Suppose that Eq. (4.31) with linear constant operators $A$ and $B$ satisfies the hypotheses:

(i) The operator $A$ is closed and has at least one regular point, the domain $D(A)$ is dense in $Y$.

(ii) The weakened Cauchy problem for Eq. (4.35) is correct on $[0, \infty)$.

(iii) $\quad D(B) \supset D(A)$ and $B u \in D(A)$, for any $u \in D(A)$.

Then on $[0, \infty)$ problem (4.31) - (4.32) has a unique solution

$$
u(t)=\left(T(t-[t])+\int_{[t]}^{t} T(t-s) B d s\right) \prod_{k=[t]}^{1}\left(T(1)+\int_{k-1}^{k} T(k-s) B d s\right) u_{0} .
$$

Consider the initial value problem

$$
\begin{gathered}
\frac{\partial u}{\partial t}=A(D) u(x, t)+f(t, u(x,[t])) \\
u(x, 0)=u_{0}(x)
\end{gathered}
$$

where $u(x, t)$ and $u_{0}(x)$ are $m$-vectors, $x=\left(x_{1}, x_{2}, \ldots, x_{N}\right) \in R^{N}$,

$$
A(D)=\sum_{|\alpha| s r} A_{\alpha} D^{\alpha}
$$

$\alpha=\left(\alpha_{1}, \alpha_{2}, \ldots, \alpha_{N}\right),|\alpha|=\alpha_{1}+\alpha_{2}+\ldots+\alpha_{N}, D^{\alpha}=D_{1}^{\alpha_{1}} \ldots D_{N}^{\alpha_{N}}, D_{k}=i \partial / \partial x_{k}(k=1,2, \ldots N)$, the coefficients $A_{\alpha}$ are given constant matrices of order $m \times m$, and the $m$-vector $f \in C^{1}\left([n, n+1) \times L^{2}\left(R^{N}\right), L^{2}\left(R^{N}\right)\right)$, $n=0,1,2, \ldots$. The number $r$ is called the order of the system. It is assumed that $u_{0} \in L^{2}\left(R^{N}\right)$, and the solutions sought are such that $u(x, t) \in L^{2}\left(R^{N}\right)$, for every $t \geq 0$. Let $\mu_{1}(s), \mu_{2}(s), \ldots, \mu_{m}(s)$ be the eigenvalues of the matrix $A(s)$. The system

$$
\frac{\partial u}{\partial t}=A(D) u
$$

is said to be parabolic by Shilov if

$$
\operatorname{Re} \mu_{j}(s) \leq-c|s|^{k}+b, j=1, \ldots, m
$$

where $h>0, c>0$, and $b$ are constants.

THEOREM 4.6. Problem (4.38) has a unique solution on $R^{N} \times[0, \infty)$ if system (4.40) is parabolic by Shilov, the index of parabolicity $h$ coincides with its order $r$, and $f \in C^{1}\left([n, n+1) \times L^{2}\left(R^{N}\right), L^{2}\left(R^{N}\right)\right)$, $n=0,1,2, \ldots$.

PROOF. For a fixed $t$ we may consider the solution $u(x, t)$ as an element of $L^{2}\left(R^{N}\right)$, and $f(t, u(x,[t])$ may be treated as an abstract function $f(t, u([t]))$ with the values in $L^{2}$. Therefore, IVP (4.38) is reduced to the abstract Cauchy problem 


$$
\frac{d u}{d t}=A u+f(t, u([t])),\left.\quad u\right|_{t=0}=u_{0} \in L^{2} .
$$

Applying to (4.40), with the initial condition $u(x, 0)=u_{0}(x)$, the Fourier transformation $F$ in $x$ produces the system of ordinary differential equations

$$
U_{t}(\omega, t)=A(\omega) U(\omega, t),
$$

with the initial condition $U(\omega, 0)=U_{0}(\omega)$, where $U(\omega, t)=F(u(x, t)), U_{0}(\omega)=F\left(u_{0}(x)\right)$, and $A(\omega)$ is a matrix with polynomial entries depending on $\omega=\left(\omega_{1}, \omega_{2}, \ldots, \omega_{N}\right)$. The solution of (4.42) is given by the formula

$$
U(\omega, t)=e^{\boldsymbol{\Lambda}(\omega)} U_{0}(\omega) .
$$

Parabolicity of (4.40) by Shilov implies that the semigroup $T(t)$ of operators of multiplication by $e^{\mathcal{A}(\omega)}$, for $t>0$, is an infinitely smooth semigroup of operators bounded in $L^{2}\left(R^{N}\right)$. Together with the requirement $h=r$, this ensures that the Cauchy problem for (4.40) is uniformly correct in $L^{2}\left(R^{N}\right)$ and all its solutions are infinitely smooth functions of $t$, for $t>0$. Since $f$ is continuously differentiable, problem (4.41) has on $[0,1)$ a unique solution

$$
u(t)=T(t) u_{0}+\int_{0}^{t} T(t-s) f\left(s, u_{0}\right) d s
$$

Denoting $u_{1}=u(1)$, we can find the solution

$$
u(t)=T(t-1) u_{1}+\int_{1}^{t} T(t-s) f\left(s, u_{1}\right) d s
$$

of $(4.41)$ on $[1,2)$ and continue this procedure successively. If $f(t, u([t]))=B u([t])$, where $B$ is a constant matrix, the solution of $(4.38)$ for $t \in[0, \infty)$ is given by (4.37). The theorem holds true if $f$ includes also the derivatives of $u(x,[t])$ in $x$ of order less than $r$, provided the initial function $u_{0}(x)$ is sufficiently smooth.

ACKNOWLEDGMENT. This research was partially supported by U.S. Army Grant DAAL03-89-G-0107, and by The University of Central Florida.

\section{REFERENCES}

1. WIENER, J. Differential equations with piecewise constant delays, in Trends in the Theory and Practice of Nonlinear Differential Equations, Lakshmikantham, V. (editor), Marcel Dekker, New York, 1983, 547-552.

2. COOKE, K. L. and WIENER, J. Retarded differential equations with piecewise constant delays, $\mathrm{J}$. Math. Anal. Appl. 99(1), (1984), 265-297.

3. SHAH, S. M. and WIENER, J. Advanced differential equations with piecewise constant argument deviations, Internat. J. Math \& Math Sci. 6(4), (1983), 671-703.

4. COOKE, K. L. and WIENER, J. Neutral differential equations with piecewise constant argument, Bolletino Unione Matematica Italiana I (1987), 321-346.

5. COOKE, K. L. and WIENER, J. An equation alternately of retarded and advanced type, Proc. Amer. Math. Soc. 99 (1987), 726-732.

6. WIENER, J. Boundary-value problems for partial differential equations with piecewise constant delay, Internat. J. Math. \& Math. Sci. 14 (1991), 301-321.

7. DEBNATH L. and MIKUSINSKI, P. Introduction to Hilbert spaces with applications, Academic Press, Boston. (1990). 
8. COOKE, K. L. and WIENER, J. Stability regions for linear equations with piecewise continuous delay, Comp. \& Math, with Appls. 12A(6), (1986), 695-701.

9. GYÖRI, I. On approximation of the solutions of delay differential equations by using piecewise constant arguments, Internat. J. Math. \& Math Sci. 14 (1991), 111-126.

10. BOROK, V. M. and ZHITOMIRSKIÜ, Y.I. The Cauchy problem for a certain class of loaded equations, Uspekhi Mat. Nauk 34 (1979), 221-222.

11. GEL'FAND, I. M. and SHILOV, G. E. Fourier transformation of rapidly increasing functions and uniqueness questions in the solution of the Cauchy problem, Uspekhi Mat. Nauk 8 (1953), 3-54. 


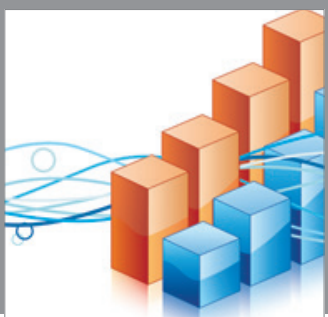

Advances in

Operations Research

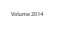

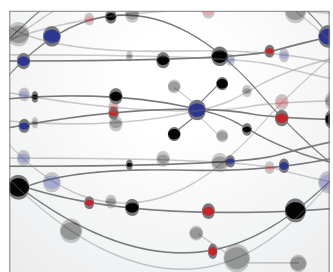

\section{The Scientific} World Journal
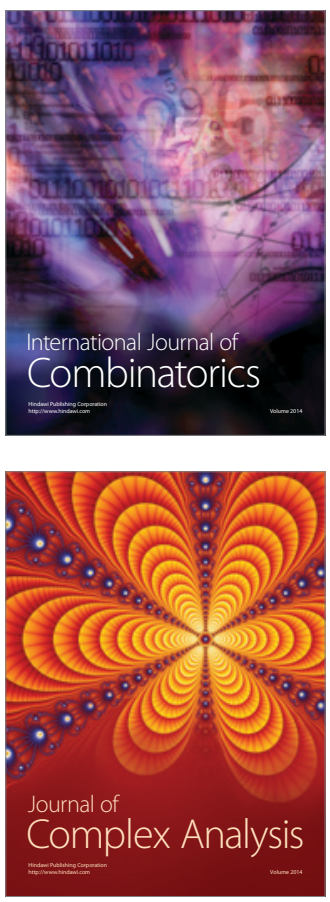

International Journal of

Mathematics and

Mathematical

Sciences
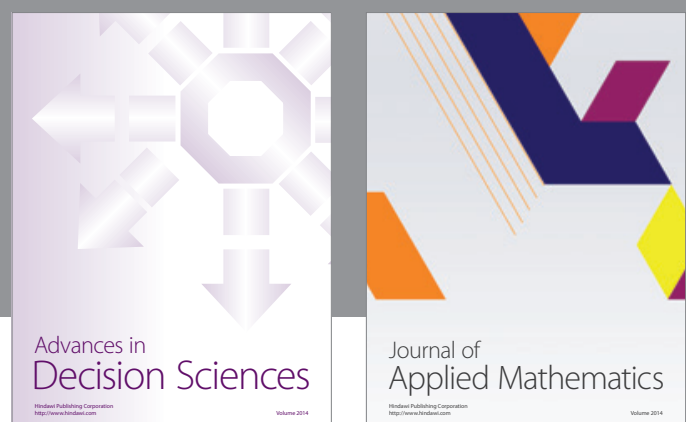

Journal of

Applied Mathematics
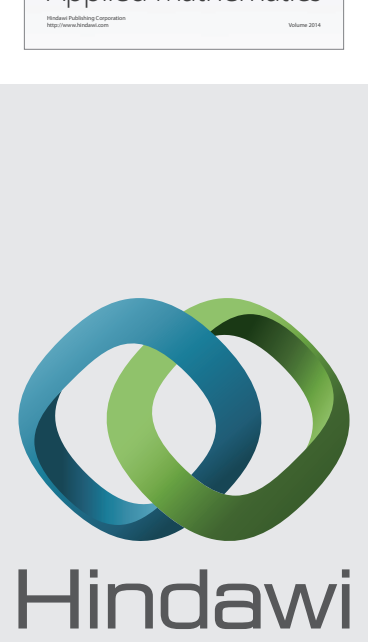

Submit your manuscripts at http://www.hindawi.com
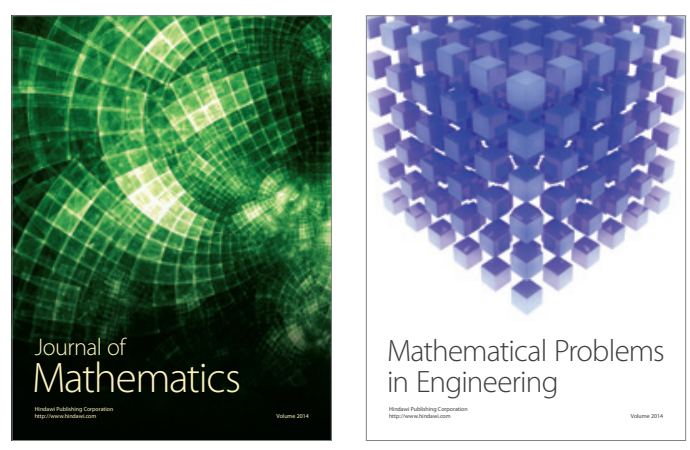

Mathematical Problems in Engineering
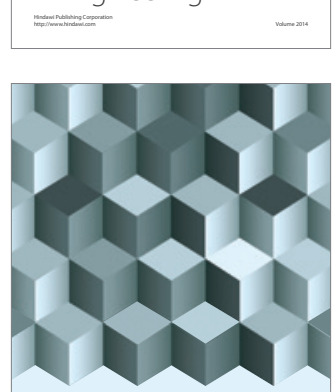

Journal of

Function Spaces
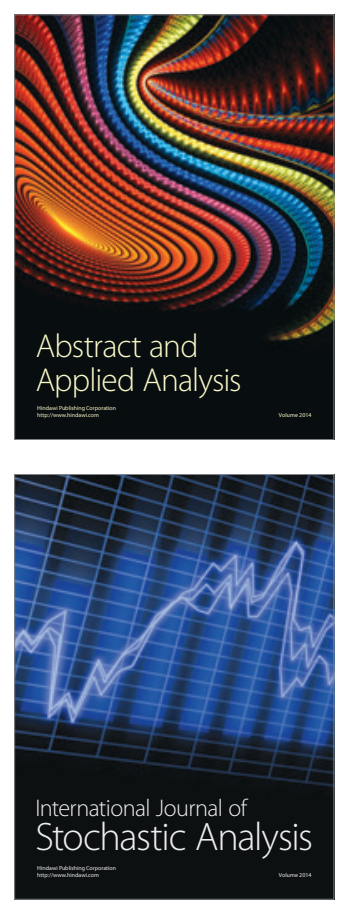

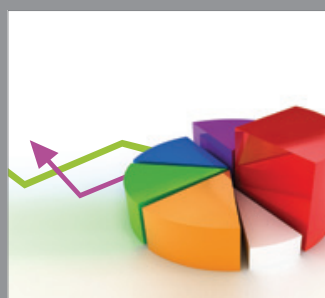

ournal of

Probability and Statistics

Promensencen
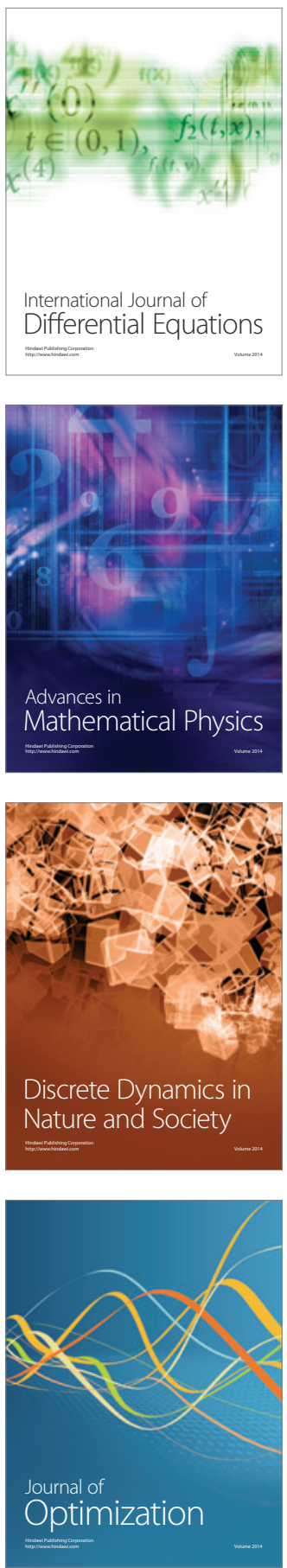